\title{
A systematic review on efficiency of microneme proteins to induce protective immunity against Toxoplasma gondii
}

\author{
Samira Dodangeh ${ }^{1,2} \cdot$ Ahmad Daryani $^{1} \cdot$ Mehdi Sharif $^{1} \cdot$ Sargis A. Aghayan $^{3} \cdot$ Abdol Satar Pagheh $^{1,2}$. \\ Shahabeddin Sarvi ${ }^{1,4} \cdot$ Fatemeh Rezaei $^{1,2}$
}

Received: 5 October 2018 / Accepted: 22 November 2018 / Published online: 24 January 2019

(C) Springer-Verlag GmbH Germany, part of Springer Nature 2019

\begin{abstract}
Toxoplasma gondii is an intracellular parasite infecting almost all warm-blooded animals. Many studies on vaccination have been performed previously, and micronemal proteins (MICs) have crucial importance in this regard. The current review aims to reveal the efficiency of MICs as target antigen, adjuvants, animal models (species/strain), T. gondii strains for challenge infection, and routes of vaccine to prevent Toxoplasma infection. A comprehensive literature search was performed on April 18, 2018, in several known databases. Studies were included when evaluating vaccines based on MIC against $T$. gondii compared to that of a control group. Two independent researchers done the search process, study choice, and data extraction. A total of 28 articles published were selected for further analysis. Among them, 57.03\% of the studies focused on MIC3 and its epitopes. SAG1 was further used in cocktail vaccines compared to other antigens. GM-CSF and Freund's complete were the predominant adjuvants used. BALB/c mice have been introduced as a proper model for lethal challenge. Virulent $T$. gondii (RH) was utilized more than other strains for challenge. Among MICs, the results of vaccination with MIC1-4, MIC6, and PLP1 demonstrated significantly strong humoral and cellular immunity, increased survival time, and reduced cyst burden in the mice. This review summarizes the latest results on MIC-based vaccines and presents that the most effective vaccination procedure is the administration of the cocktail vaccines. Our survey can serve as a basis for further studies to develop more efficient novel vaccines against $T$. gondii for animals and humans.
\end{abstract}

Keywords DNA vaccine $\cdot$ Microneme proteins $\cdot$ Recombinant vaccine $\cdot$ Toxoplasmosis $\cdot$ Vaccine candidates

\section{Introduction}

Toxoplasma gondii (T. gondii), an apicomplaxan protozoan parasite that causes toxoplasmosis, is an opportunistic pathogen invading broad host range, including humans and nearly all warm-blooded animals worldwide [1]. Toxoplasmosis is a

Shahabeddin Sarvi

shahabesarvi@yahoo.com

1 Toxoplasmosis Research Center, Mazandaran University of Medical Sciences, Sari, Iran

2 Student Research Committee, School of Medicine, Mazandaran University of Medical Sciences, Sari, Iran

3 Laboratory of Zoology, Research Institute of Biology, Yerevan State University, Yerevan, Republic of Armenia

4 Department of Medical Parasitology, School of Medicine, Mazandaran University of Medical Sciences, $18 \mathrm{~km}$ of Khazar Abad Road, PO Box 48175-1665, Sari, Iran life-threatening and significant infectious disease which also results to economic losses in animal production, especially in pigs, sheep, and goats [2]. Moreover, T. gondii-infected meat products are a major source of human infection [3]. T. gondii, as an opportunistic infection factor, can be fatal for patients with suppressed immune systems, especially for those with acquired immunodeficiency syndrome (AIDS) [4], undergoing organ transplantation [5], and patients with malignant tumors receiving cancer therapy [6]. In these patients, rapid multiplication of cytolytic tachyzoites destroys the nervous tissues and causes the pathological changes associated with toxoplasmic encephalitis (TE) [7]. In addition, toxoplasmosis is a significant biological agent influencing human prenatal and postnatal care because $T$. gondii infection during pregnancy can lead to maternal-fetal vertical transmission causing miscarriage, premature birth, severe cognitive impairment, or ocular complications (blindness) in the fetus [8]. Despite increasing studies concerning the biology of the parasite, there is a lack of chemotherapeutic agents to limit its proliferation 
during the rapid replication stages (tachyzoite), and there are no available therapeutic agents to eliminate the cyst stages from the infected host [9]. On the other hand, development of a vaccine against $T$. gondii would be particularly valuable for controlling human and animal toxoplasmosis. According to studies, excretory-secretory antigens (ESAs) of T. gondii have been observed as important component during the process of invasion and replication of tachyzoites within host cells and are considered to be one of the important targets of host immune responses [10].

In recent years, several trials on DNA-based and proteinbased vaccines have focused on $T$. gondii antigens belonging to several major protein families, such as the glycosylphosphatidylinositol (GPI)-anchored proteins named SAG (surface antigens), SRS (SAG1-related sequences), and SUSA (SAG-unrelated surface antigens), rhoptry antigens (ROPs), dense granule antigens (GRAs), and micronemal proteins (MICs) [11-14].

Among these, micronemal proteins (MICs) are small apical organelles, including several adhesion proteins, that are secreted during early contact of the parasite's apical end with the host plasma membrane. The protozoa enters the host cell using its own actin-myosin system $[15,16]$. The parasite is able to enter wide range of host cell types due to being homologous in modular structures from most MICs to ligand domains or adhesion domains from eukaryotic proteins, such as chitin binding-like (CBL), epidermal growth factor-like (EGF), thrombospondin type-1 repeat (TSR), and microneme adhesive repeat (MAR) domains. MICs are not only essential for attachment to the host cell's entry but are also crucial for parasite gliding motion, as their cytoplasmic domains can bind to aldolase that is connected to parasite actin-myosin motor, the basis for active invasion [17]. Recently, the MIC complex was shown to play a predominant and important role in virulence and pathogenicity [18]. Currently, at least 19 types of MICs were known, including MIC1-MIC12, AMA1, M2AP, PLP1, ROM1, SPATR, SUB1, and TLN4 [19]. An increasing number of studies on MICs have showed them as potent antigen targets and vaccine candidates to induce powerful immune responses against toxoplasmosis. The current review aims to provide a more comprehensive understanding of the T. gondii MICs (TgMICs), adjuvants, animal models (species/ strain), T. gondii strains for challenge infection, and routes of vaccine used to prevent and control $T$. gondii infection through a systematic search on complete interventions aimed at reducing toxoplasmosis.

\section{Methods}

This review was planned and prepared according to the specified process in the PRISMA (Preferred Reporting Items for Systematic Reviews and Meta-Analyses) statement [20]. All of our methods are publicly available in the CAMARADES website (Collaborative Approach to Meta-Analysis and Review of Animal Data from Experimental Studies; http: //www.camarades.info/index_files/chickenyolkSRt.pdf).

\section{Search process}

A comprehensive literature search about effect of immunization with TgMICs on animal models was performed in PubMed, Web of Science, Proquest, Scopus, Science Direct, and Embase in April 18, 2018, by 2 independent authors (SD and $\mathrm{ShS}$ ). The search was initiated from the first described vaccination with MICs in 2003 using a combination of keywords as follows: toxoplasmosis, Toxoplasma, vaccine, vaccination, immunization, protection, MIC, microneme, and micronemal. This was complemented by using further searches from bibliographies in related primary and review articles.

\section{Inclusion criteria}

A paper was included if it fulfilled the following criteria: (1) the study used every form of DNA-vaccine or protein-vaccine or both based on MIC against $T$. gondii; (2) the research evaluated vaccination on animal models challenged with tachyzoites or cysts of $T$. gondii; (3) literature was published in English language and accessible in full text; (4) the article had appropriate control groups; and (5) the research has high score.

\section{Exclusion criteria}

After reviewing articles, studies with the following criteria were excluded: (1) the study did not report any survival time and parasite burden; (2) the research did not have enough information; (3) literature had no control group; and (4) the article did not achieve the minimum score, 6 , for its quality.

\section{Study choice}

Two authors (SD and ASP) independently assessed the titles and abstracts of the articles. Full texts of relevant studies were reviewed independently to ensure their eligibility for inclusion into the systematic review. Each disagreement was resolved with discussion or upon review by another researcher (AD).

\section{Data extraction}

Data on gene used, adjuvant, route of vaccine, inoculated vaccine dose, animal model, number and strain of injected parasites, challenge route, immunology, survival, and parasite burden were independently summarized by two reviewers using a standardized data collection form. In addition, we 
extracted bibliographic data, such as authors and year of publication.

\section{Assessment of quality}

The quality of all the included studies was assessed using the Systematic Review Center for Laboratory Animal Experimentation (SYRCLE), a tool based on the Cochrane Collaboration Risk of Bias tool [21]. The SYRCLE statement contains 10 items, and one of them concerning random housing of animals was removed. Therefore, nine items remained for selection, performance, detection, attrition, and reporting biases. The maximum score is 9. Higher SYRCLE scores show better quality article.

\section{Results}

\section{Number of included studies}

In total, 28 articles (DNA vaccine 23; protein vaccine 4; DNA and protein vaccine 1), published from 2003 to 2017 [13, 22-48], were included in this review. In total, the SYRCLE scores of the included studies ranged from 6 to 9 , with a low of 7.9. This emphasizes a low risk of bias (Tables 1, 2). Among them, approximately $57.03 \%$ of the studies focused on MIC3 and its epitopes. Figure 1 briefly demonstrates the search process for this systematic review.

\section{DNA vaccine}

Out of the 23 studies conducted on DNA vaccine, 12 (54.5\%) focused on MIC3 and its epitopes (epidermal growth factorlike (EGF) domains and the Lectin-like domain), and multiple other molecules, such as $\mathrm{MIC} 1, \mathrm{MIC} 2$, perforin-like protein (PLP1), MIC4, MIC6, MIC8, $\alpha$-chain of MIC11, MIC13, M2AP, and AMA, were also surveyed, either alone or in combination (11 studies single, 2 cocktail, and 9 both single and cocktail), for their vaccine potential at different efficacy levels. Moreover, SAG1 was further used in cocktail vaccines compared to other antigens, including ROP18, GRAs, and other MICs (Table 1).

\section{Protein vaccine}

There were 5 protein vaccines in the included studies, and 3 of these 5 studies focused on MIC3 (60\%). In addition, there was no study on cocktail vaccine regarding the combination of microneme protein with ROPs and GRAs.

\section{Adjuvants}

Various adjuvants have been used to improve vaccine potency, including bacteria-derived adjuvants (LPS, CpG motifs, cholera toxin A2/B), cytokines (GM-CSF, IL-18, IL-12), emulsions (Freund's complete and incomplete), and particles (gold particles). Among these, GM-CSF (2/11 studies) and Freund's complete (2/11 studies) were employed more than the other mentioned adjuvants.

\section{Route of vaccine delivery}

The most used route for vaccine delivery to animal models was intramuscular (22 from 28 studies), and other ways of vaccination used include the subcutaneous, oral, gene gun into the abdomen, footpad, and intranasal routes. Recently, intranasal immunization with MIC8 demonstrated $100 \%$ protection, whereas intramuscular vaccination resulted in only $60 \%$ survival [47].

\section{Animal models}

The recommended vaccines were based on animal models (mice). The three most utilized mouse models for vaccination were BALB/c, Kunming, and C57BL/6. However, a few studies also used CBA/J, ICR, and Swiss mice as animal models. Among these, $\mathrm{BALB} / \mathrm{c}$ mice have been employed as a proper model for lethal challenge tests with highly virulent $(\mathrm{RH}$ strain) $T$. gondii. In contrast, C57BL/6 mice exhibit a low rate of survival with even a low dose of a non-virulent strain of T. gondii. According to Dautu et al., $20 \%$ of the BALB/c mice immunized with M2AP survived for 30 days, whereas none of the C57BL/6 mice survived for the same period [24]. In addition, detected IFN- $\gamma$ in mice immunized with MIC2 and AMA1 in BALB/c (55.89 \pm 8.5 and $126.0 \pm 33)$ was more compared to C57BL/6 (35.00 \pm 5.0 and $126.0 \pm 33)$ [24].

\section{Challenge}

For surveying efficiency of vaccines, around 2-4 weeks after immunization, the mice were challenged with $T$. gondii infection. To study the survival rates, the mice were infected intraperitoneally ( 16 studies) or orally ( 6 studies) or intragastrically ( 2 studies) with $10^{2}-10^{4}$ tachyzoites or 10-80 cysts (rarely) from different parasite strains. Virulent $T$. gondii $(\mathrm{RH})$ was utilized more than the other strains (ME49, Beverley, Trousseau, and PRU). Subsequently, mortality was checked daily and survival time was recorded. To examine protective immunity, avirulent or less virulent strains, such as SSI119, $76 \mathrm{~K}$, PRU, and ME49, were administered orally (7 studies) or intraperitoneally ( 2 studies) or intragastrically (1 study) with $20-70$ cysts per mouse. However, in the study of Gong et al., the mice were challenged with $1 \times 10^{2}$ tachyzoites of $T$. gondii 


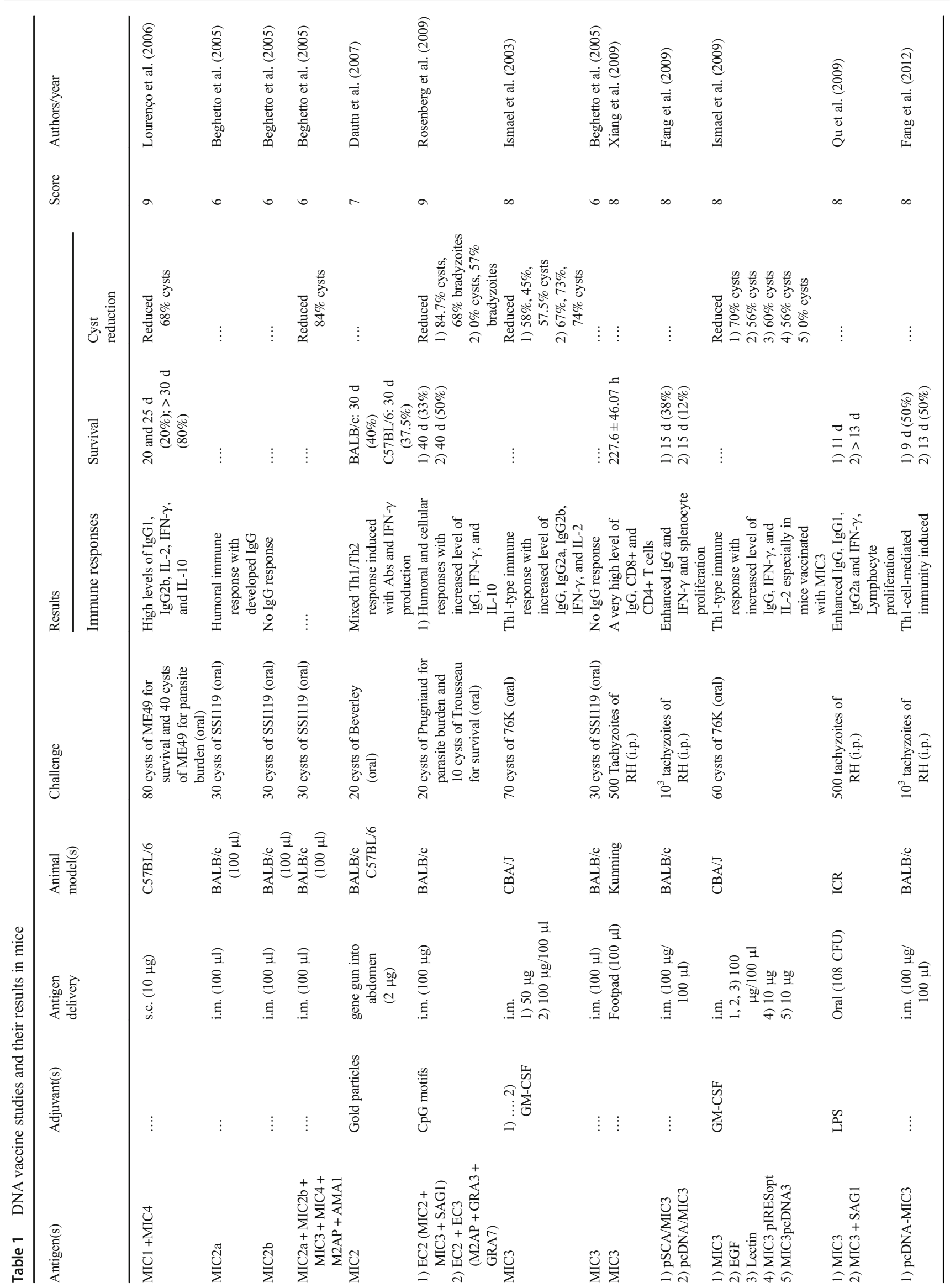




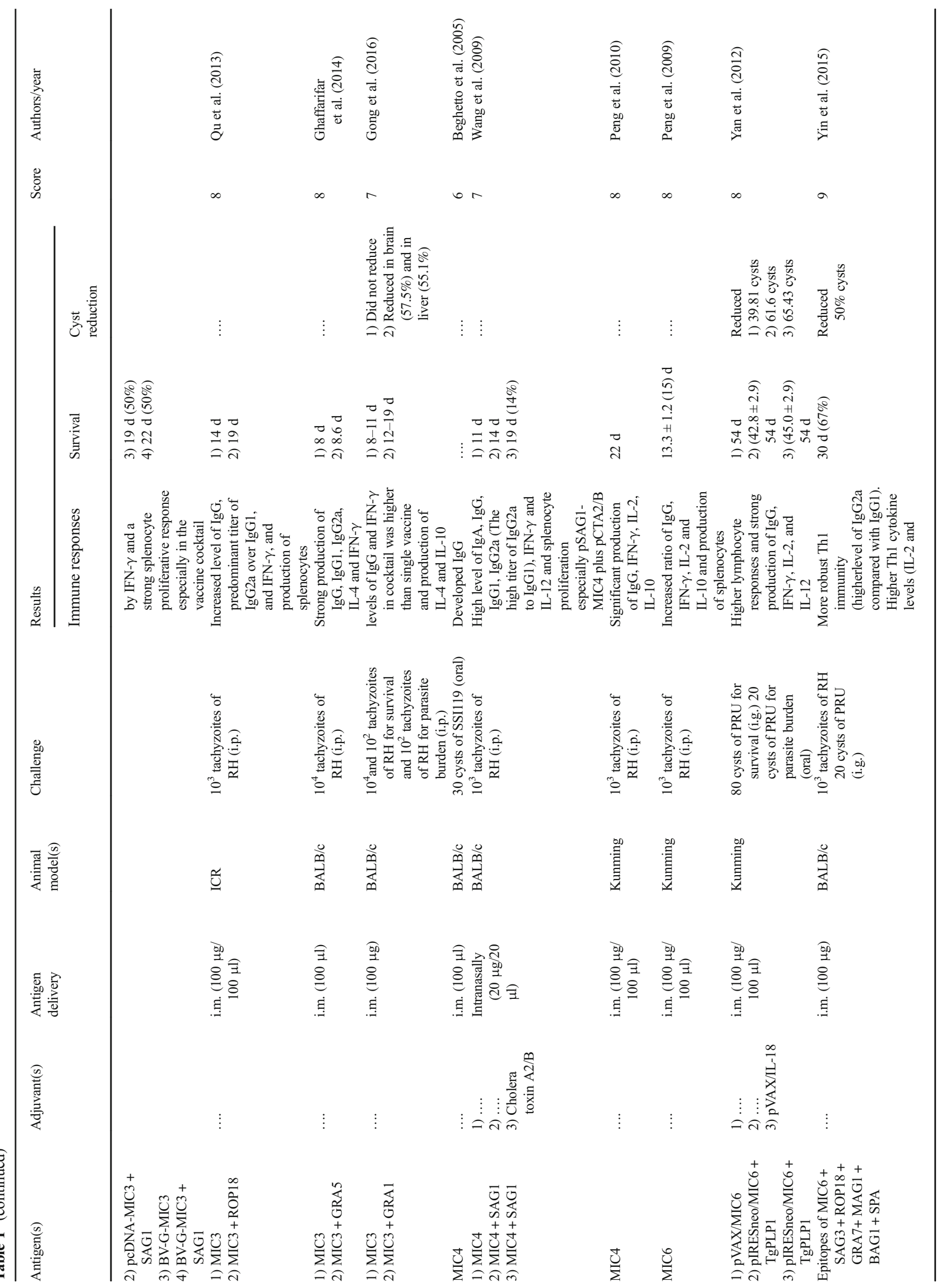




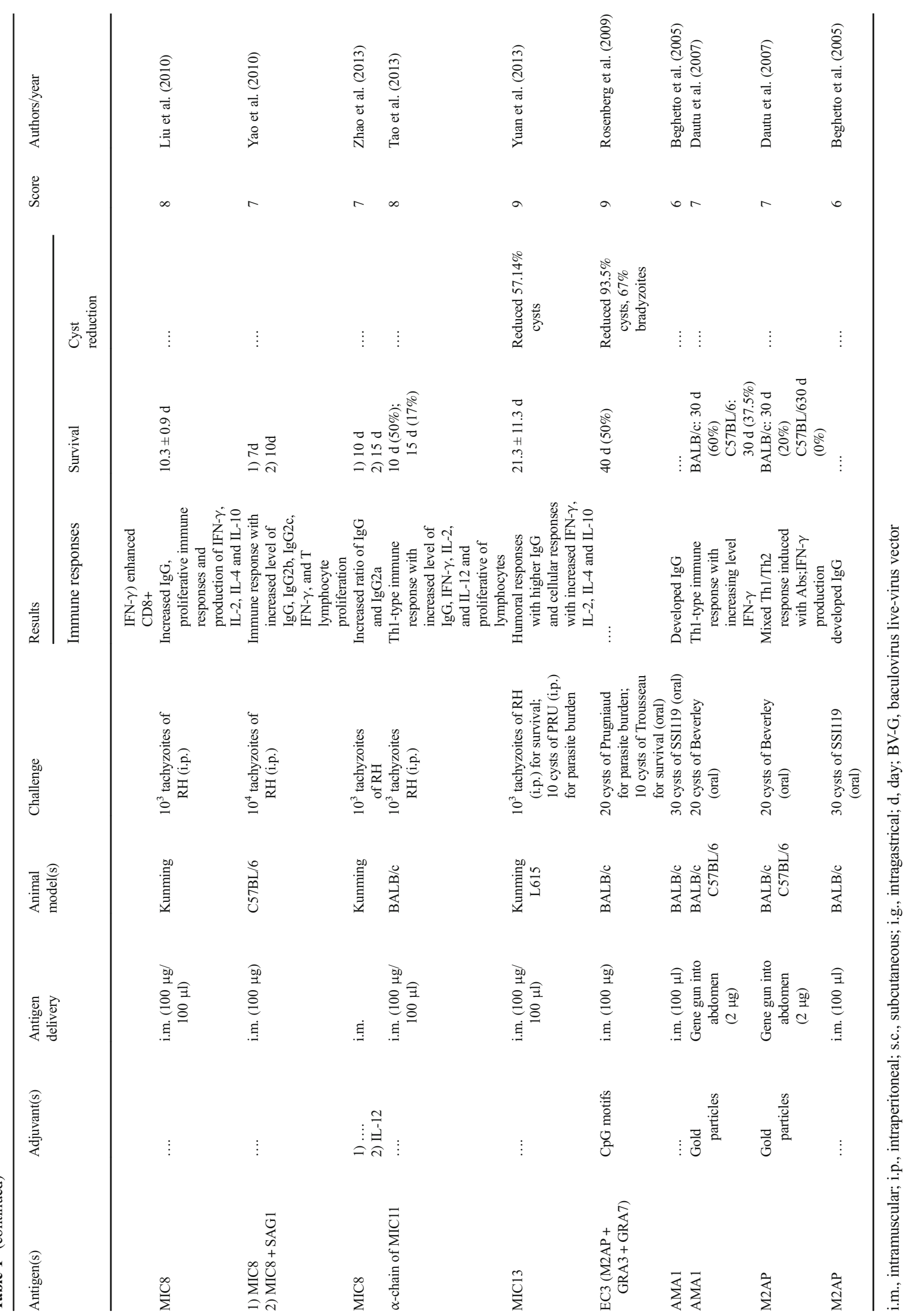




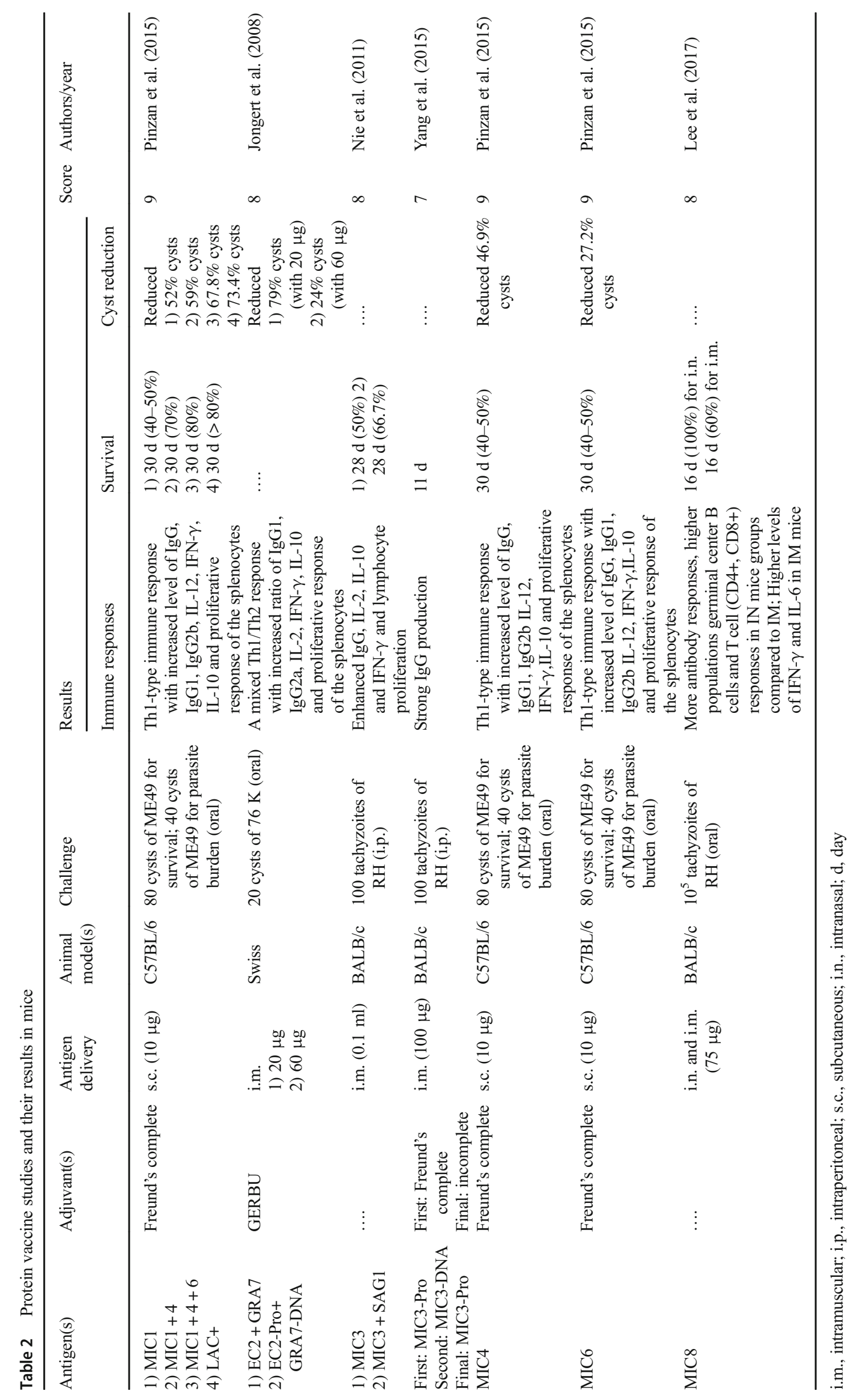


Fig. 1 Flowchart describing the study design process
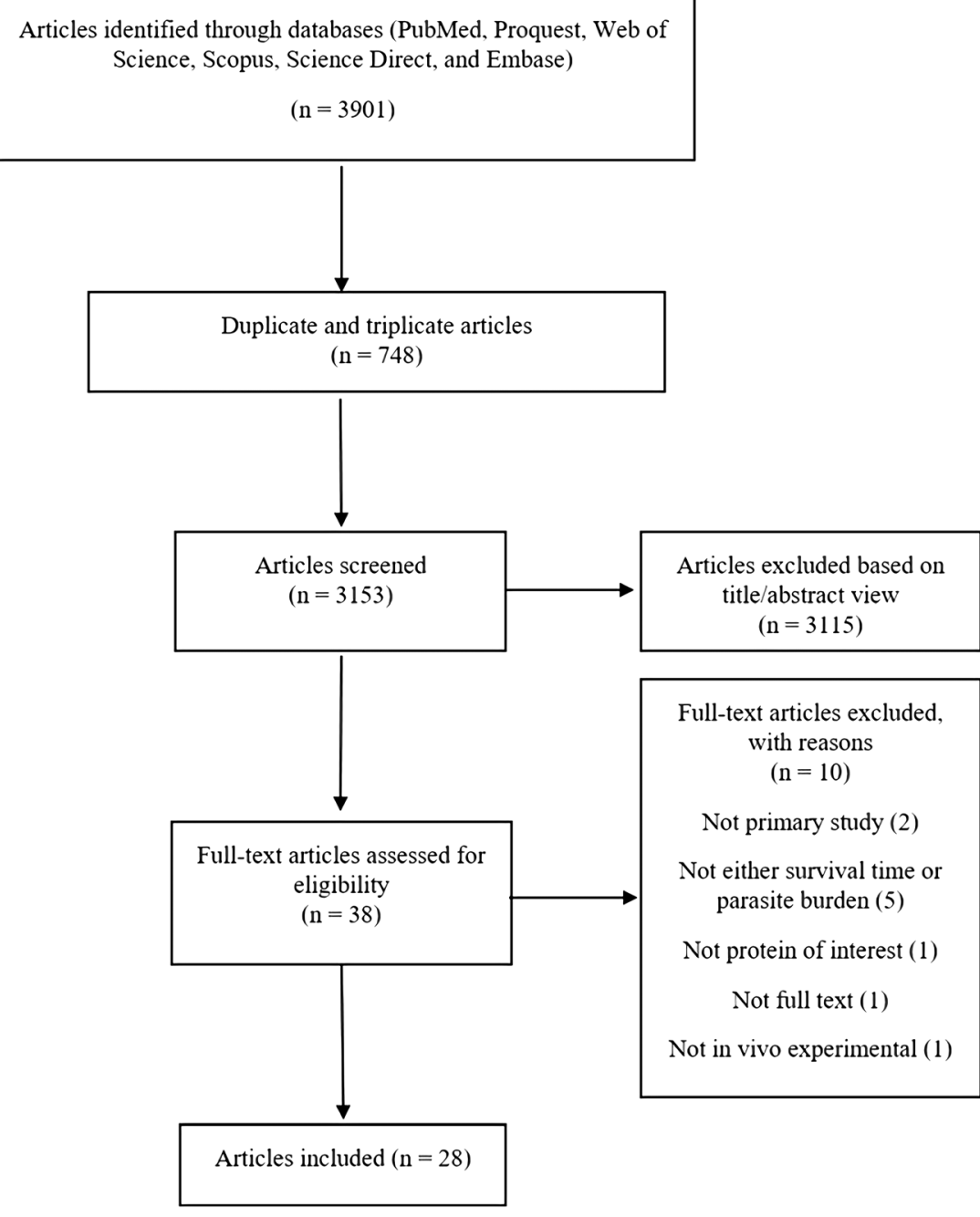

Full-text articles excluded, with reasons $(\mathrm{n}=10)$

Not primary study (2)

Not either survival time or parasite burden (5)

Not protein of interest (1)

Not full text (1)

Not in vivo experimental (1)
RH strain intraperitoneally [34]. Reduction in brain cyst burden of the chronic model was then recorded.

\section{Immune response}

All sera were analyzed by ELISA to evaluate the levels of anti-T. gondii antibodies. Significantly high levels of total $\mathrm{IgG}$ and its subclasses were detected in the sera of mice vaccinated with plasmid-MICs, especially after the third vaccination (Tables 1, 2).

With regard to the findings, mice immunized with cocktail vaccines, such as SAG1-MIC3 (nearly $\operatorname{IgG}=0.8$ compared to 0.2 with OD $630 \mathrm{~nm}$ ) [45], MIC6-PLP1 (nearly $\mathrm{IgG}=1.8$ compared to 1.3 with OD $450 \mathrm{~nm}$ ) [38], and MIC1-4-6 (nearly $\operatorname{IgG}=0.6$ compared to 0.3 with OD $490 \mathrm{~nm}$ ) [13], presented higher levels of specific antibodies than those immunized with single vaccines $(P<0.05)$.

Additionally, to assess the cellular immune responses in the vaccinated mice, lymphocyte proliferation and cytokine production in splenocyte suspensions were measured. The lymphocyte proliferation response was considerably higher in all immunized mice than the control groups $(P<0.05)$. Significantly higher levels of IL-2, IL-10, IL-12, and IFN- $\gamma$ were produced in splenocyte cultures from vaccinated mice compared with the control groups. However, there were no considerable differences in the production of IL-4 between the immunized and the non-immunized groups (Tables 1, 2). Results of multiple studies demonstrate that the cellular immune responses induced by the multi-antigenic vaccines were better than those induced by single genes.

For example, spleen cells from mice immunized with MIC1-4-6 [13] produced twice as much IL-12 than the cells from mice vaccinated with single-component MIC proteins. In addition, significant production of IFN- $\gamma(1136 \pm 152)$ and IL-12 (845 \pm 37$)$ was observed from spleen cells in mice immunized with pMIC4-SAG1 compared to mice immunized with single-gene SAG1 $(658 \pm 117$ and $432 \pm 42)$ or MIC4 $(632 \pm 96$ and $415 \pm 23)$ [35]. Qu and collaborators also observed higher levels of IFN- $\gamma$ from spleen cell cultures in mice immunized with MIC3-ROP18 $(849 \pm 86)$ compared with 
single-gene ROP18 $(427 \pm 40)$ or MIC3 $(346 \pm 31)$ [32]. Further, the mice immunized with the vaccine cocktail MIC3-SAG1 produced a stronger splenocyte proliferative response than the mice immunized with MIC3 or SAG1 with mean SI (stimulation index) of 15 and 10, respectively [31].

\section{Protective effect in immunized mice}

Mice immunized with 2-50 $\mathrm{gg} / \mu \mathrm{l}$ MIC1, MIC2, MIC4, MIC6, AMA1, and M2AP (single or cocktail) showed extension of their survival time by a rough maximum of thirty days. Though many studies utilized $100 \mu \mathrm{g} / \mu \mathrm{l}$ antigen, it is important to mention that the survival time of Kunming mice challenged with $80 \mathrm{~T}$. gondii cysts of the PRU strain survived for up to 54 days following the injection of $100 \mu \mathrm{g} / \mu \mathrm{l}$ MIC6 [38]. Among several single antigens employed for vaccination under similar conditions (animal model, route and dose of vaccine, and inoculation parasite for challenge), MIC3, MIC4 (22 days) [27, 36], and MIC13 (21 days) [44] improved the survival time significantly compared to the others (approximately 10 days). Additionally, combination vaccines, such as SAG1-MIC3 [30], ROP18-MIC3 [32], MIC3 + GRA1 [34], SAG1-MIC4 [35], SAG1-MIC8 [41], and MIC1-4-6 [13], demonstrated increased survival compared to single antigens.

Some studies also assayed the reduction in brain cyst burden following vaccination. Parasite burdens in the brain reduced from $0 \%$ (mice vaccinated with $10 \mu \mathrm{g} / \mu \mathrm{l} \mathrm{MIC3}$ ) to 93.5\% (mice vaccinated with $100 \mu \mathrm{g} / \mu \mathrm{l}$ EC3 (GRA3 + GRA7 + M2AP)) [25, 29]. According to the study by Pinzan and collaborators, LAC+, native complex (MIC1 + MIC4) isolated from STAg (Soluble Toxoplasma Antigen), reduced the parasite burden to $73.4 \%$ compared to individual component, namely, MIC1 (52\%) [13].

\section{Discussion}

The aim of the present systematic review was to survey DNAbased and protein-based vaccines encoding $T$. gondii MICs to demonstrate their capacity for inducing immune responses (humoral and cellular), survival times, and protection in animal models after challenge with $T$. gondii strains. Despite substantial advances in the research on immune responses that occur after infection with $T$. gondii, there is currently only one commercially available vaccine, "Toxovax," which has been used to decrease the abortion incidence in sheep for veterinary uses, but it did not prevent the formation of tissue cysts [49]. However, it has several disadvantages and cannot be utilized for humans because such vaccines may regain virulence and even provoke iatrogenic infection [50]. Thus, the development of a safe and effective vaccine would be globally valuable to prevent both recrudescent and fetal infections in immunecompromised patients and pregnant women [51]. In other words, ideal vaccines against $T$. gondii should be able to eliminate the parasite and subsequently prevent cyst formation and maternal-fetal transmission. Since there is no ideal vaccine for complete elimination of the parasite, studies should focus on appropriate antigens to increase survival time and decrease cyst number in animal models as primary endpoints.

Therefore, the findings of the present systematic review encourage and support better studies in the future to produce the next-generation vaccines against $T$. gondii. Both recombinant proteins and DNA vaccines have been shown to protect hosts against intracellular parasites.

According to this systematic review, among the MIC-based vaccine candidates, MIC3 was the most frequently applied as an important vaccine and elicits strong, long-lasting humoral and cellular immunities [26-34]. This protein has a potent affinity to host cells, and its receptor-binding site is closely associated with the N-terminal chitin binding-like (CBL) domain [52]. Moreover, MIC3 was found to be expressed during 3 parasite life stages, including tachyzoite, bradyzoite, and sporozoite [19], although microneme proteins usually are not highly expressed during the sporozoite stage. Humans are usually infected by ingesting oocysts released from cat feces or by eating meat from infected animals containing long-lived tissue cysts [53]. Thus, immunization with MIC3 would generate a specific immune response that could avoid the release of tachyzoites from cysts (bradyzoites) and mature oocysts (sporozoites). Bioinformatics analysis demonstrated that MIC3 has a great immunogenicity and antigenicity index [26]. Therefore, it is considered as a potential candidate for vaccine development.

Previous studies have displayed that single-gene vaccines have been encouraged but are only able to induce partial immune responses against $T$. gondii [26]. Recently, increasing investigations have focused on multi-antigenic or cocktail vaccines. Multiple studies indicate that either DNA vaccine or recombinant vaccine in cocktail form, such as MIC3SAG1, MIC3-GRA1, MIC3-ROP18, MIC4-SAG1, MIC6PLP1, MIC8-SAG1, and MIC1-4-6, induces a stronger immunogenicity against $T$. gondii challenge compared to singlegene vaccines [13, 30, 32-34, 38, 41]. Given its complex life cycle involving numerous hosts with various forms of proteins to express and diversity of invasion pathways, $T$. gondii represents a very vast degree of antigenic variability. Further, accumulating evidences display that immunization with stage-specific antigens elicits stage-limited protection against toxoplasmosis. Therefore, it is important to develop multiantigen vaccines in connection with multi-stage in order to conquer the deficiency of utilizing single antigens as vaccine candidates.

Epitope-based vaccine design is a novel method that primarily recognizes sequential epitopes [54]. The efficacy of epitope-based vaccines is extremely dependent on the exact identification of conformational B cell and T cell epitopes. Yin 
et al. surveyed epitopes of several antigens derived from tachyzoite, bradyzoite, and sporozoite of $T$. gondii, including SAG3 (101-144), ROP18 (347-396), MIC6 (288-347), GRA7 (182-224), MAG (158-125), BAG1 (156-211), and SPA (142-200), by priming with DNA vaccine and boosting with adenovirus-vectored vaccine, and this immunization proved a potential protect in mice challenged with $T$. gondii [39]. Compared with common vaccines, epitope-based vaccines have many advantages. Given that they do not contain inactivated pathogens, such vaccines are safer, thereby entirely eliminating any possible threat of infection [55].

We found that vaccination efficacy is particularly dependent on the utilized adjuvant, route of administration, strain and life cycle stage of the parasite employed, and the challenge infection. Moreover, the animal model (species/strain) used is also crucial for developing vaccines for humans.

Usually, vaccines do not have sufficient immunological potency and need to be combined with proper adjuvants to increase their effectiveness. Various adjuvants have been used as part of the vaccine formulation, including Freund's complete adjuvant (FCA), Freund's incomplete adjuvant (FIA), gold particles, GM-CSF, LPS, GERBU, cholera toxin, CpG, IL-12, and IL-18. Interestingly, immunization with Th1inducing gold particles (as an adjuvant) incorporating a few $(2 \mu \mathrm{g})$ MIC2 or M2AP or AMA could increase the survival of $\mathrm{C} 57 \mathrm{BL} / 6$ and $\mathrm{BALB} / \mathrm{c}$ mice challenged with the strain Beverley [24].

In addition to the use of adjuvants in vaccines, efficiency may also be increased by changing the vaccination route as intradermal inoculation is shown to be more effective than the intramuscular or intraperitoneal routes [56]. However, few recent studies employing the intranasal route have demonstrated the potential of particularly targeting mucosal immunity [35]. The reason why large number of studies employ intramuscular vaccination might be that the musculature is full of cells which leads to rapid and abundant expression of the related protein. Interestingly, DNA vaccines were administrated by different routes, such as intramuscular injection or a DNA particle delivery system, Gene Gun. Unlike intramuscular injection, which requires micrograms $(\mu \mathrm{g})$ of plasmid DNA and several doses, Gene Gun route requires lesser levels of plasmid DNA for inducing the same immune response [24].

The level of protection afforded by specific vaccines is also shown to be dependent on the murine model utilized. For instance, though M2AP protected BALB/c mice with regard to survival time, it could not protect C57BL/6 mice [24]. Similarly, GRA6 provided partial protection against T. gondii in BALB/c mice but not in Kunming mice [57]. As formerly infected female BALB/c mice are capable of inhibiting congenital transmission completely even if reinfected during pregnancy, this model represents a gold standard for designing appropriate vaccine strategies.
Thus, firstly immunization should be carried out on inbred mouse strains, especially BALB/c and $\mathrm{C} 57 \mathrm{BL} / 6$ or $\mathrm{C} 3 \mathrm{H} / \mathrm{HeN}$; in addition, the vaccine efficacy should also be examined in outbred mice, since these are more similar to animal and human population [58]. Although in a study, HiszczyńskaSawicka and collaborators examined immune responses including IgG1 and IgG2 antibodies and production of IFN- $\gamma$ using MIC3 DNA vaccine on sheep, it would be better for future studies to evaluate survival time and parasite burden in such animal models [59].

Susceptibility to toxoplasmosis varies with the diverse routes of challenge used for the strain variety of $T$. gondii [60]. According to the findings, in approximately $77 \%$ of the studies, the mice were challenged orally for examination of parasite burden in brain cysts. Challenge protocols of the studies depend on the aim of vaccination. For prevention of acquired toxoplasmosis (using food consumption), oral challenge route is better than other routes, whereas in transplacental form, non-oral routes, such as intraperitoneal or subcutaneous, seem to be more suitable.

In addition, different $T$. gondii strains are applied for challenge in vivo. Approximately $81.7 \%$ of the studies utilized virulent $T$. gondii $\mathrm{RH}$ to generate an acute model in mice. It is presently proven that $T$. gondii strains vary in virulence in mammals [61]. Particularly, Brazilian Toxoplasma strains are more virulent compared to strains of the European types I/II/ III, and a number of other Toxoplasma strains are appearing through congenital toxoplasmosis in human and animal reservoirs globally [58]. In a study of Carneiro and collaborators, a total of 27 T. gondii strains were isolated from newborns with congenital toxoplasmosis in the state of Minas Gerais, Brazil, tested by PCR-RFLP approach. Among them, 14 isolates $(54 \%)$ were identified as intermediate virulence, 10 isolates $(38 \%)$ as virulent, and only 2 isolates $(8 \%)$ as nonvirulent [62]. In addition, 20 Toxoplasma gondii strains isolated from animals and humans in Brazil by Ferreira and collaborators [63] were analyzed through two RAPD-PCR and SSR-PCR techniques. They showed that $85 \%$ of the studied strains had a certain degree of virulence, whereas only $15 \%$ of the strains were non-virulent. These results are in contrast with strains surveyed in the United States and Europe, where most strains are non-virulent [64]. Thus, it is important to utilize genotypes originating from different parts of the world in the immunization measures to ensure cross-virulence immunization against T. gondii [58].

It is a consensus opinion that cell-mediated immune responses, especially associated with CD8 T lymphocytes producing IFN- $\gamma$, are the main mediator of immunity against toxoplasmosis, making it a promising strategy for vaccination investigations. In addition, CD4 T cells play an important role in inducing immune responses and produce interleukin-2 (IL2) for the development of CD8 T cells. Nevertheless, numerous vaccine studies have also indicated that a broad range of 
immune responses requiring elements of humoral immunity with antibodies provide the best protection against infection [65]. Consequently, numerous studies have surveyed whether immune responses to MICs and other candidate antigens, such as (GPI)-anchored protein (SAG, SRS, and SUSA), GRA, ROP, either singly or in a cocktail, induce protection against challenge by evaluating the survival time and brain cyst burden [66]. DNA and protein vaccines have been demonstrated as potent methods for the induction of particular cellular and humoral immune responses. Protein vaccines are demonstrated to induce primarily humoral immunity and, thus, are extremely efficient for controlling infections that could be antagonized with neutralizing antibodies. DNA vaccines, however, induce primarily potent cytotoxic $\mathrm{T}$ cell responses and have been showed to have limited ability in overall protection, especially when utilized in primates [58]. In a review, Foroutan and collaborators reported that a heterologous prime-boost strategy, especially a DNA vaccine as a priming and a protein-based vaccine for booster, induces more cellular and humoral immune responses than the homologous prime-boost [67].

Furthermore, studies on MICs have made much progress, and their compounds and functions are now becoming apparent. With regard to results, among MICs, MIC1-4, MIC6, and PLP1 prolonged the survival time in the mice by an average of 38 days and reduced the parasite burden by an average of $72 \%$ $[13,38]$.

Therefore, these evidence suggest that the MIC-based vaccines presented in this systematic review are promising and can be a worthy starting point to develop an effective and novel vaccine against $T$. gondii, for use in animals and humans. Recent advances in experimental and bioinformatics tools will aid in the selection of better vaccine candidates.

Another approach for toxoplasmosis control is the vaccines that directly target $T$. gondii transmission. Thus, it is important to design a vaccine providing potential contribution to decrease the infection rates, prevent parasite development, and reduce toxoplasmosis by inhibiting the transmission. $T$. gondii transmission-blocking vaccines (TBVs) are the tools to reduce infection in the family Felidae (domestic cats and their relatives) and toxoplasmosis transmission, by inducing the immunity that breaks the life cycle of the parasite between humans and cats.

\section{Conclusion}

Multi-epitope synthetic peptide vaccines delivering multistage MICs (MIC3, MIC4, and MIC13) can induce significantly higher immunogenicity. Also, the most successful vaccination procedure was the administration of the cocktail vaccines, since vaccinated animal models had the highest survival time and the lowest tissue cyst burden in the brain. According to the present systematic review, the strongest protection against toxoplasmosis was induced in mice by the mixture of surface antigens (SAG1) or other ESAs (ROP18 and GRA7) combined with MIC proteins. In addition, evaluation in other animal host species, containing those in which a vaccination may have veterinary usage, should aim in defining the role that this vaccination may play in controlling toxoplasmosis. Moreover, protection in animal models should be enhanced using gold particles (as an adjuvant). It is worth noting that bioinformatics and in silico approaches will facilitate production of novel potential vaccines against Toxoplasma gondii.

Acknowledgments The authors would like to acknowledge Miss Tooran Nayeri and Miss Zahra Hosseini Nejad for their kind collaboration and the Student Research Committee, Mazandaran University of Medical Sciences, Sari, Iran, for their support to this study. This study was financially supported by the Vice-Chancellor of Mazandaran University of Medical Sciences (Project Number: 10221).

\section{Compliance with ethical standards}

Declaration of interests The authors report that they have no conflicts of interest. The authors alone are responsible for the content and writing of the paper.

Publisher's note Springer Nature remains neutral with regard to jurisdictional claims in published maps and institutional affiliations.

\section{References}

1. Dubey JP (2008) The history of Toxoplasma gondii-the first 100 years. J Eukaryot Microbiol 55(6):467-475

2. Buxton D (1998) Protozoan infections (Toxoplasma gondii, Neospora caninum and Sarcocystis spp.) in sheep and goats: recent advances. Vet Res 29(3):289-310

3. Sarvi S, Daryani A, Rahimi MT, Aarabi M, Shokri A, Ahmadpour E, Mizani A, Sharif M (2015) Cattle toxoplasmosis in Iran: a systematic review and meta-analysis. Asian Pac J Trop Med 8(2):120-126

4. Ahmadpour E, Daryani A, Sharif M, Sarvi S, Aarabi M, Mizani A, Rahimi MT, Shokri A (2014) Toxoplasmosis in immunocompromised patients in Iran: a systematic review and meta-analysis. The Journal of Infection in Developing Countries 8(12):1503-1510

5. Escuissato DL, de Aguiar RO, Gasparetto EL, Müller NL (2004) Disseminated toxoplasmosis after bone marrow transplantation: high-resolution CT appearance. J Thorac Imaging 19(3):207-209

6. Israelski DM, Remington JS (1993) Toxoplasmosis in patients with cancer. Clin Infect Dis 17(Supplement 2):S423-S435

7. Kasper L, Buzoni-Gatel D (1998) Some opportunistic parasitic infections in AIDS: candidiasis, pneumocystosis, cryptosporidiosis, toxoplasmosis. Parasitol Today 14(4):150-156

8. Mizani A, Alipour A, Sharif M, Sarvi S, Amouei A, Shokri A, Rahimi M-T, Hosseini SA, Daryani A (2017) Toxoplasmosis seroprevalence in Iranian women and risk factors of the disease: a systematic review and meta-analysis. Tropical Medicine and Health 45(1):7

9. Montazeri M, Sharif M, Sarvi S, Mehrzadi S, Ahmadpour E, Daryani A (2017) A systematic review of in vitro and in vivo activities of anti-toxoplasma drugs and compounds (2006-2016). Front Microbiol 8 
10. Eldin HME, Kamel HH, Badawy AF, Shash LS (2015) A comparative study between excretory/secretory and autoclaved vaccines against RH strain of Toxoplasma gondii in murine models. J Parasit Dis 39(3):526-535

11. Wu X-N, Lin J, Lin X, Chen J, Chen Z-L, Lin J-Y (2012) Multicomponent DNA vaccine-encoding Toxoplasma gondii GRA1 and SAG1 primes: anti-Toxoplasma immune response in mice. Parasitol Res 111(5):2001-2009

12. Parthasarathy S, Fong MY, Ramaswamy K, Lau YL (2013) Protective immune response in BALB/c mice induced by DNA vaccine of the ROP8 gene of Toxoplasma gondii. The American Journal of Tropical Medicine and Hygiene 88(5):883-887

13. Pinzan CF, Sardinha-Silva A, Almeida F, Lai L, Lopes CD, Lourenço EV, Panunto-Castelo A, Matthews S, Roque-Barreira MC (2015) Vaccination with recombinant microneme proteins confers protection against experimental toxoplasmosis in mice. PLoS One 10(11):e0143087

14. Macêdo Junior AG, Cunha Junior JP, Cardoso THS, Silva MV, M Santiago F, Silva JS, Pirovani CP, Silva DAO, Mineo JR, Mineo TWP (2013) SAG2A protein from Toxoplasma gondii interacts with both innate and adaptive immune compartments of infected hosts. Parasit Vectors 6:163

15. Dobrowolski JM, Carruthers VB, Sibley LD (1997) Participation of myosin in gliding motility and host cell invasion by Toxoplasma gondii. Mol Microbiol 26(1):163-173

16. Meissner M, Schluter D, Soldati D (2002) Role of Toxoplasma gondii myosin $\mathrm{A}$ in powering parasite gliding and host cell invasion. Science 298(5594):837-840

17. Liu Q, Li FC, Zhou CX, Zhu XQ (2017) Research advances in interactions related to Toxoplasma gondii microneme proteins. Exp Parasitol 176:89-98

18. Cérède O, Dubremetz JF, Soête M, Deslée D, Vial H, Bout D, Lebrun M (2005) Synergistic role of micronemal proteins in Toxoplasma gondii virulence. J Exp Med 201(3):453-463

19. Wang Y, Yin H (2015) Research advances in microneme protein 3 of Toxoplasma gondii. Parasit Vectors 8(1):384

20. Moher D, Liberati A, Tetzlaff J, Altman DG, Group P (2009) Preferred reporting items for systematic reviews and meta-analyses: the PRISMA statement. PLoS Med 6(7):e1000097

21. Hooijmans CR, Rovers MM, de Vries RB, Leenaars M, RitskesHoitinga M, Langendam MW (2014) SYRCLE's risk of bias tool for animal studies. BMC Med Res Methodol 14:43

22. Lourenço EV, Bernardes ES, Silva NM, Mineo JR, PanuntoCastelo A, Roque-Barreira M-C (2006) Immunization with MIC1 and MIC4 induces protective immunity against Toxoplasma gondii. Microbes Infect 8(5):1244-1251

23. Beghetto E, Nielsen HV, Del Porto P, Buffolano W, Guglietta S, Felici F, Petersen E, Gargano N (2005) A combination of antigenic regions of Toxoplasma gondii microneme proteins induces protective immunity against oral infection with parasite cysts. J Infect Dis 191(4):637-645

24. Dautu G, Munyaka B, Carmen G, Zhang G, Omata Y, Xuenan X, Igarashi M (2007) Toxoplasma gondii: DNA vaccination with genes encoding antigens MIC2, M2AP, AMA1 and BAG1 and evaluation of their immunogenic potential. Exp Parasitol 116(3): 273-282

25. Rosenberg C, De Craeye S, Jongert E, Gargano N, Beghetto E, Del Porto P, Vorup-Jensen T, Petersen E (2009) Induction of partial protection against infection with Toxoplasma gondii genotype II by DNA vaccination with recombinant chimeric tachyzoite antigens. Vaccine 27(18):2489-2498

26. Ismael AB, Sekkai D, Collin C, Bout D, Mévélec M-N (2003) The MIC3 gene of Toxoplasma gondii is a novel potent vaccine candidate against toxoplasmosis. Infect Immun 71(11):6222-6228

27. Xiang W, Qiong Z, Li-peng L, Kui T, Jian-wu G, Heng-ping S (2009) The location of invasion-related protein MIC3 of
Toxoplasma gondii and protective effect of its DNA vaccine in mice. Vet Parasitol 166:1):1-1):7

28. Fang R, Nie H, Wang Z, Tu P, Zhou D, Wang L, He L, Zhou Y, Zhao J (2009) Protective immune response in BALB/c mice induced by a suicidal DNA vaccine of the MIC3 gene of Toxoplasma gondii. Vet Parasitol 164(2):134-140

29. Ismael $\mathrm{AB}$, Hedhli $\mathrm{D}$, Cérède $\mathrm{O}$, Lebrun $\mathrm{M}$, Dimier-Poisson I, Mévélec M-N (2009) Further analysis of protection induced by the MIC3 DNA vaccine against T. gondii: CD4 and CD8 T cells are the major effectors of the MIC3 DNA vaccine-induced protection, both lectin-like and EGF-like domains of MIC3 conferred protection. Vaccine 27(22):2959-2966

30. Qu D, Yu H, Wang S, Cai W, Du A (2009) Induction of protective immunity by multiantigenic DNA vaccine delivered in attenuated Salmonella typhimurium against Toxoplasma gondii infection in mice. Vet Parasitol 166(3):220-227

31. Fang R, Feng H, Hu M, Khan MK, Wang L, Zhou Y, Zhao J (2012) Evaluation of immune responses induced by SAG1 and MIC3 vaccine cocktails against Toxoplasma gondii. Vet Parasitol 187(1): $140-146$

32. Qu D, Han J, Du A (2013) Evaluation of protective effect of multiantigenic DNA vaccine encoding MIC3 and ROP18 antigen segments of Toxoplasma gondii in mice. Parasitol Res 112(7): 2593-2599

33. Ghaffarifar F, Naserifar R, Jafari Madrak M (2014) Eukaryotic plasmids with Toxoplasma gondii dense granule antigen (GRA 5) and microneme 3 (MIC3) genes as a cocktail DNA vaccine and evaluation of immune responses in $\mathrm{BALB} / \mathrm{C}$ mice. $\mathrm{J}$ Clin $\mathrm{Med}$ Genom 3 (121):2

34. Gong P, Cao L, Guo Y, Dong H, Yuan S, Yao X, Ren W, Yao L, Xu Z, Sun Q (2016) Toxoplasma gondii: protective immunity induced by a DNA vaccine expressing GRA1 and MIC3 against toxoplasmosis in BALB/c mice. Exp Parasitol 166:131-136

35. Wang H, He S, Yao Y, Cong H, Zhao H, Li T, Zhu X-Q (2009) Toxoplasma gondii: protective effect of an intranasal SAG1 and MIC4 DNA vaccine in mice. Exp Parasitol 122(3):226-232

36. Peng G, Yuan Z, Zhou D, He X, Yan C, Yin C, He Y, Lin R, Song H, Zhu X (2010) Sequence variation in Toxoplasma gondii MIC4 gene and protective effect of an MIC4 DNA vaccine in a murine model against toxoplasmosis. J Anim Vet Adv 9:1463-1468

37. Peng G-H, Yuan Z-G, Zhou D-H, He X-H, Liu M-M, Yan C, Yin CC, He Y, Lin R-Q, Zhu X-Q (2009) Toxoplasma gondii microneme protein 6 (MIC6) is a potential vaccine candidate against toxoplasmosis in mice. Vaccine 27(47):6570-6574

38. Yan H-K, Yuan Z-G, Song H-Q, Petersen E, Zhou Y, Ren D, Zhou D-H, Li H-X, Lin R-Q, Yang G-L (2012) Vaccination with a DNA vaccine coding perforin-like protein 1 (TgPLP1) and MIC6 induces significant protective immunity against Toxoplasma gondii. Clin Vaccine Immunol:CVI. 05578-05511

39. Yin H, Zhao L, Wang T, Zhou H, He S, Cong H (2015) A Toxoplasma gondii vaccine encoding multistage antigens in conjunction with ubiquitin confers protective immunity to BALB/c mice against parasite infection. Parasit Vectors 8(1):498

40. Liu M, Yuan Z, Peng G, Zhou D, He X, Yan C, Yin C, He Y, Lin R, Song H (2010) Toxoplasma gondii microneme protein 8 (MIC8) is a potential vaccine candidate against toxoplasmosis. Parasitol Res 106(5):1079-1084

41. Yao Y, He S, Wang H, Zhou H, Zhao H, Li T, Xue M, Zhu X (2010) Protective immunity induced in mice by multiantigenic DNA vaccine with genes encoding SAG1 and MIC8 of Toxoplasma gondii. Zhongguo ji sheng chong xue yu ji sheng chong bing za zhi = Chinese Journal of Parasitology \& Parasitic Diseases 28(2):81-88

42. Zhao H, Huang F, Guo J, Tan G (2013) Evaluation on the immune response induced by DNA vaccine encoding MIC8 co-immunized with IL-12 genetic adjuvant against Toxoplasma gondii infection. Zhongguo ji sheng chong xue yu ji sheng chong bing za zhi $=$ 
Chinese Journal of Parasitology \& Parasitic Diseases 31(4):284 289

43. Tao Q, Fang R, Zhang W, Wang Y, Cheng J, Li Y, Fang K, Khan MK, Hu M, Zhou Y (2013) Protective immunity induced by a DNA vaccine-encoding Toxoplasma gondii microneme protein 11 against acute toxoplasmosis in BALB/c mice. Parasitol Res 112(8):2871-2877

44. Yuan Z-G, Ren D, Zhou D-H, Zhang X-X, Petersen E, Li X-Z, Zhou Y, Yang G-L, Zhu X-Q (2013) Evaluation of protective effect of pVAX-TgMIC13 plasmid against acute and chronic Toxoplasma gondii infection in a murine model. Vaccine 31(31):3135-3139

45. Jongert E, Verhelst D, Abady M, Petersen E, Gargano N (2008) Protective Th1 immune responses against chronic toxoplasmosis induced by a protein-protein vaccine combination but not by its DNA-protein counterpart. Vaccine 26(41):5289-5295

46. Nie H, Fang R, Xiong B-Q, Wang L-X, Hu M, Zhou Y-Q, Zhao J-L (2011) Immunogenicity and protective efficacy of two recombinant pseudorabies viruses expressing Toxoplasma gondii SAG1 and MIC3 proteins. Vet Parasitol 181(2):215-221

47. Yang D, Liu J, Hao P, Wang J, Lei T, Shan D, Liu Q (2015) MIC3, a novel cross-protective antigen expressed in Toxoplasma gondii and Neospora caninum. Parasitol Res 114(10):3791-3799

48. Lee S, Kim A, Lee D, Rubino I, Choi H, Quan F (2017) Protection induced by virus-like particles containing Toxoplasma gondii microneme protein 8 against highly virulent $\mathrm{RH}$ strain of Toxoplasma gondii infection. PLoS One 12(4):e0175644

49. Buxton D, Thomson K, Maley S, Wright S, Bos H (1991) Vaccination of sheep with a live incomplete strain (S48) of Toxoplasma gondii and their immunity to challenge when pregnant. The Veterinary Record 129(5):89-93

50. Buxton D, Innes E (1995) A commercial vaccine for ovine toxoplasmosis. Parasitology 110(S1):S11-S16

51. Innes EA (2010) Vaccination against Toxoplasma gondii: an increasing priority for collaborative research? Expert Review of Vaccines 9(10):1117-1119

52. Cérède O, Dubremetz JF, Bout D, Lebrun M (2002) The Toxoplasma gondii protein $\mathrm{MIC} 3$ requires pro-peptide cleavage and dimerization to function as adhesin. EMBO J 21(11):2526-2536

53. Lepage AC, Buzoni-Gatel D, Bout DT, Kasper LH (1998) Gutderived intraepithelial lymphocytes induce long term immunity against Toxoplasma gondii. J Immunol 161(9):4902-4908

54. Frank R (2002) The SPOT-synthesis technique: synthetic peptide arrays on membrane supports-principles and applications. J Immunol Methods 267(1):13-26

55. Wang Y, Wang G, Cai J, Yin H (2016) Review on the identification and role of Toxoplasma gondii antigenic epitopes. Parasitol Res 115(2):459-468
56. Mohamed RM, Aosai F, Chen M, Mun H-S, Norose K, Belal US, Piao L-X, Yano A (2003) Induction of protective immunity by DNA vaccination with Toxoplasma gondii HSP70, HSP30 and SAG1 genes. Vaccine 21(21):2852-2861

57. Sun X-M, Zou J, AA ES, Yan W-C, Liu X-Y, Suo X, Wang H, Chen Q-J (2011) DNA vaccination with a gene encoding Toxoplasma gondii GRA6 induces partial protection against toxoplasmosis in $\mathrm{BALB} / \mathrm{c}$ mice. Parasit Vectors 4(1):213

58. Jongert E, Roberts CW, Gargano N, Förster-Waldl E, Petersen E (2009) Vaccines against Toxoplasma gondii: challenges and opportunities. Memorias do Instituto Oswaldo Cruz 104(2):252-266

59. Hiszczyńska-Sawicka E, Li H, Xu J, Akhtar M, Holec-Gąsior L, Kur J, Bickerstaffe R, Stankiewicz M (2012) Induction of immune responses in sheep by vaccination with liposome-entrapped DNA complexes encoding Toxoplasma gondii MIC3 gene. Pol J Vet Sci 15(1):3-9

60. Henriquez FL, Woods S, Cong H, McLeod R, Roberts CW (2010) Immunogenetics of Toxoplasma gondii informs vaccine design. Trends Parasitol 26(11):550-555

61. Boyle JP, Rajasekar B, Saeij JP, Ajioka JW, Berriman M, Paulsen I, Roos DS, Sibley LD, White MW, Boothroyd JC (2006) Just one cross appears capable of dramatically altering the population biology of a eukaryotic pathogen like Toxoplasma gondii. Proc Natl Acad Sci 103(27):10514-10519

62. Carneiro ACAV, Andrade GMQ, Costa JGL, Pinheiro BV, Vasconcelos-Santos DV, Ferreira AM, Su C, Januario JN, Vitor RWA (2013) Genetic characterization of Toxoplasma gondii revealed highly diverse genotypes for isolates from newborns with congenital toxoplasmosis in southeastern Brazil. J Clin Microbiol 51(3):901-907

63. Ferreira ADM, Vitor RWA, Gazzinelli RT, Melo MN (2006) Genetic analysis of natural recombinant Brazilian Toxoplasma gondii strains by multilocus PCR-RFLP. Infect Genet Evol 6(1): 22-31

64. Howe DK, Honore S, Derouin F, Sibley LD (1997) Determination of genotypes of Toxoplasma gondii strains isolated from patients with toxoplasmosis. J Clin Microbiol 35(6):1411-1414

65. Mordue DG, Hunter CA Innate immunity toToxoplasma gondiichapter 24

66. Kur J, Holec-Gąsior L, Hiszczyńska-Sawicka E (2009) Current status of toxoplasmosis vaccine development. Expert Review of Vaccines 8(6):791-808

67. Foroutan M, Zaki L, Ghaffarifar F (2018) Recent progress in microneme-based vaccines development against Toxoplasma gondii. Clinical and Experimental Vaccine Research 7(2):93-103 